\title{
Asteroid 2867 Steins
}

\section{Photometric properties from OSIRIS/Rosetta and ground-based visible observations ${ }^{\star}$}

\author{
L. Jorda ${ }^{1}$, P. L. Lamy ${ }^{1}$, G. Faury ${ }^{1}$, P. Weissman ${ }^{2}$, M. A. Barucci ${ }^{3}$, S. Fornasier ${ }^{3,4}$, S. Lowry ${ }^{5}$, \\ I. Toth ${ }^{1,6}$, and M. Küppers ${ }^{7}$
} \author{
38 rue Frédéric Joliot-Curie, 13388 Marseille Cedex 13, France \\ e-mail: laurent.jorda@oamp.fr \\ 2 Jet Propulsion Laboratory, 4800 Oak Grove Drive, MS 183-301, Pasadena, CA 91109, USA \\ 3 LESIA, Observatoire de Paris, 92195 Meudon Principal Cedex, France \\ Université de Paris 7 Denis Diderot, France \\ School of Mathematics and Physics, Queen's University, Belfast, UK \\ 6 Konkoly Observatory, PO Box 67, 1525, Hungary \\ 7 Max-Planck-Institut für Sonnensystemforschung, Germany
}

Received 5 November 2007 / Accepted 25 May 2008

\section{ABSTRACT}

\begin{abstract}
Context. Asteroid 2867 Steins is the first target of the Rosetta space mission with a flyby scheduled in September 2008. Aims. An early characterization is needed to optimize the flyby parameters and the science operations and to maximize the scientific return. The aim of this article is to characterize the photometric properties of this asteroid.

Methods. The asteroid was imaged with the Narrow Angle Camera (NAC) of the Optical, Spectroscopic, and Infrared Remote Imaging System (OSIRIS) during the cruise phase of the Rosetta spacecraft, at a phase angle of $41.7^{\circ}$ significantly larger than achievable from Earth. It was continuously monitored over $\sim 24 \mathrm{~h}$ (on 11 March 2006), thus covering four rotational periods, at a temporal cadence of 6 min. An accurate photometric calibration was obtained from the observations of a solar analog star, 16 Cyg B.

Results. The light curve in the $R$ photometric band of the Johnson-Kron-Cousins system has a mean value $R\left(1,1, \alpha=41.7^{\circ}\right)=14.13 \pm$ 0.03 and an amplitude of $0.25 \pm 0.04$ mag. The periodicity was analyzed with different techniques yielding a mean value of the synodic rotational period of $6.054 \pm 0.003 \mathrm{~h}$. By combining with ground-based observations obtained at different phase angles, the phase function is constructed and characterized by a linear part having a phase coefficient $\beta=0.025 \pm 0.001 \mathrm{mag} / \mathrm{deg}$ and a mean value $R(1,1,0)=13.10 \pm 0.04$. In terms of the $H-G$ formalism, the best fit photometric values are $G=0.35 \pm 0.05$ and $H=12.84 \pm$ 0.07 , but the resulting opposition surge of $0.25 \mathrm{mag}$, although typical of E-type asteroids, is not really constrained because of the lack of data at phase angles below $7^{\circ}$. Altogether the photometric properties of asteroid 2867 Steins (phase function, color and albedo) indicate that it is a somewhat extreme E-type object, although it is known that this quite small population exhibits at least three different surface mineralogies.
\end{abstract}

Key words. minor planets, asteroids - techniques: photometric

\section{Introduction}

The Rosetta spacecraft was successfully launched on 2 March 2004 and is now on its way to a rendez-vous with comet 67P/Churyumov-Gerasimenko in May 2014 at a heliocentric distance of 4.5 AU. During its journey to the comet, the spacecraft will fly by two main belt asteroids: 2867 Steins and 21 Lutetia, selected after detailed studies of the candidates of all possible mission scenarios (Barucci et al. 2005). Nominally the fly-by of asteroid Steins will take place on 5 September 2008, at a relative velocity of $8.6 \mathrm{~km} \mathrm{~s}^{-1}$ and a closest approach of $800 \mathrm{~km}$. The geometry of the encounter is such that the spacecraft will approach Steins at a constant phase angle of $38^{\circ}$. The Narrow Angle Camera (NAC) of the Optical, Spectroscopic, and Infrared Remote Imaging System (OSIRIS)

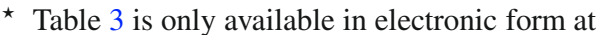
http: //www . aanda.org will start monitoring the unresolved asteroid two weeks before closest approach (ca) to secure several light curves to refine the determination of its rotational state, and to constrain its global shape. The phase angle will decrease to nearly $0^{\circ}$ slightly before ca, and then increase rapidly, reaching $45^{\circ}$ at ca, and culminating at $140^{\circ}$ during the outbound trajectory. The rotation of the asteroid will ensure that $100 \%$ of its surface will be seen, albeit at different spatial resolutions. It is expected that $62 \%$ will be imaged at a scale better than $200 \mathrm{~m} /$ pixel. At closest approach, the asteroid will extend over $\sim 300$ pixels across the field-of-view of the NAC, and the spatial resolution (2 pixels) will reach $30 \mathrm{~m}$. The resolved images will be used to retrieve the shape and photometric properties of Steins, and to build mineralogic maps using different filters. As presently planned, the NAC will obtain about 450 images, and its companion Wide Angle Camera (WAC) about 300 images. 
As for all targets of space missions, an a priori knowledge of these "terra incognita" is of crucial importance for the preparation and optimization of the operations of the spacecraft and its instruments so as to maximize the scientific return. We have already completed a detailed characterization of the nucleus of $67 \mathrm{P} /$ Churyumov-Gerasimenko thanks to timely observations with the Hubble and Spitzer space telescopes (Lamy et al. 2006, 2007), and this has been crucial for European Space Agency's assessment of the feasibility of the mission. Further observations were obtained when the comet was at 5.6 AU from the sun and inactive, using European Southern Observatory's $3.5 \mathrm{~m}$ New Technology Telescope, Chile (Lowry et al. 2006). We now turn our attention to the first target asteroid, 2867 Steins in a series of four companion articles.

- This present article (I) presents a refined analysis of the data obtained with the OSIRIS-NAC aboard the Rosetta spacecraft at a phase angle unreachable from Earth, and discusses the photometric properties (color, phase function).

- Article II (Lamy et al. 2008a) is devoted to multi-telescope visible observations, shape reconstruction and rotational state.

- Article III (Lamy et al. 2008b) reports on Spitzer space telescope observations in the infrared and focuses on the size, albedo and thermal properties.

- Article IV (Barucci et al. 2008) reports on the thermal emissivity, and discusses mineralogical composition and classification.

The small main belt asteroid 2867 Steins was discovered on 4 November, 1969 by Chernykh at Nauchnyj beveled (Crimea) and named in memory of Karlis Augustovich Šteins (1911-1983), director of the Latvian University's Astronomical Observatory. It is only recently that various efforts have been made to characterize this object (Hicks et al. 2004; Barucci et al. 2005; Weissman et al. 2007). In particular, Fornasier et al. (2006) indirectly determined its diameter using the empirical relationship between albedo and polarization. The resulting high albedo $(0.45 \pm 0.1)$ then implies a diameter of approximately $4.6 \mathrm{~km}$, and classifies 2867 Steins as an E-type asteroid, a rare class of asteroid with properties similar to the enstatite meteorites as already proposed by Barucci et al. (2005) and confirmed by Barucci et al. (2008), Article IV, and by Weissman et al. (2007).

In this article, we present an in-depth analysis of the images of 2867 Steins obtained with the OSIRIS narrow angle camera (NAC) aboard the Rosetta spacecraft during its long cruise phase in interplanetary space. This data set is unique in terms of both the continuous coverage over $24 \mathrm{~h}$ and of the large phase angle of $41.7^{\circ}$, significantly larger than the maximum value of $30^{\circ}$ achievable from Earth (presently available observations are however limited to $27^{\circ}$ ). A first analysis of the OSIRIS-NAC data has been reported by Küppers et al. (2007). They focused on the periodicity and they derived a synodic period of $6.052 \pm$ $0.007 \mathrm{~h}$ from a relative light curve without quantifying the errors. They also derived a magnitude $V(1,1, \alpha)=14.68$ and an absolute magnitude $H=13.05 \pm 0.03$ with the assumption that the slope parameter $G$ is equal to 0.15 . In this article, we present the observations and data reduction, and we extensively describe the calibration procedure, based on the direct photometry of the solar analog 16 Cyg B, as well as the photometric uncertainties. We then present the calibrated light curve, and briefly discuss the rotational properties based on the application of different techniques. We review the color data to transform all available absolute magnitudes to the common Johnson-Kron-Cousins $R$ band. Combining these magnitudes obtained at different phase angles

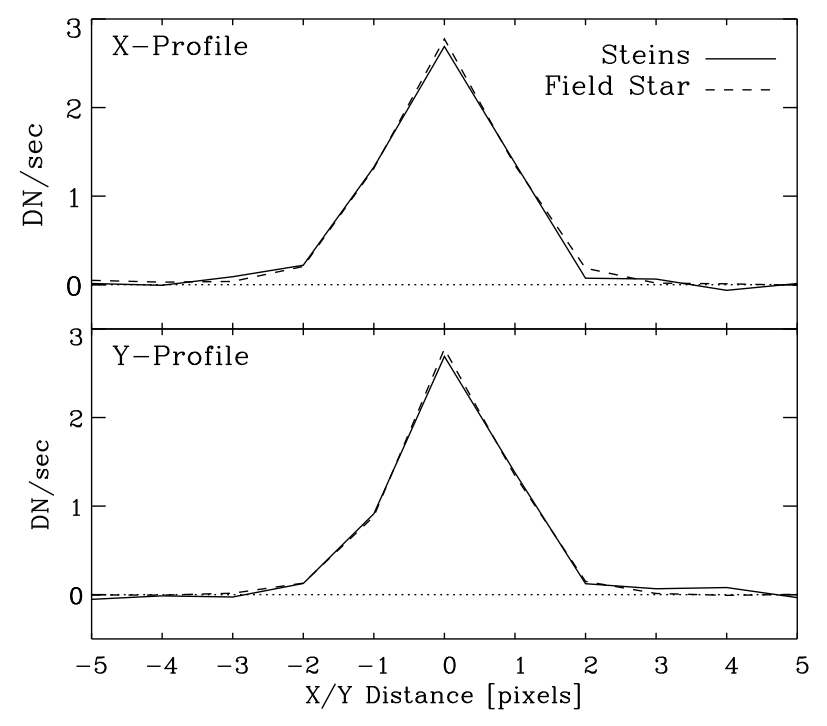

Fig. 1. Profiles of the point spread function of the OSIRIS narrow angle camera with the $F 21$ "clear" filter as obtained from the asteroid and from a star on the same image.

allow us to determine the phase function of 2867 Steins, and we discuss the implications for its classification.

\section{Observations}

The narrow angle camera (NAC) is the high-resolution camera of the OSIRIS imaging system (Keller et al. 2007) aboard ROSETTA. The NAC is a three-mirror anastigmat telescope with an unobstructed aperture of $90 \mathrm{~mm}$ and a focal length of $717 \mathrm{~mm}$ $(\mathrm{f} / \mathrm{D}=8)$. In addition to its original optical concept for a planetary camera, the NAC implements a novel all silicon carbide mechanical design where the mirrors and telescope structure are made of the same material SiC (Dohlen et al. 1996; Calvel et al. 1999). It is equipped with a dual filter wheel and an E2V 42-40 $2048^{2}$ pixels back-side, back-thinned CCD. The pixel size of $13.5 \mu \mathrm{m}$ corresponds to a pixel field-of-view of 3.88 arcsec. The NAC offers an excellent point source sensitivity: the optical point spread function has a $F W H M$ of $8.3 \operatorname{arcsec}(2.15$ pixel): it is adequatly sampled by the CCD detector. Figure 1 displays the $X$ and $Y$ profiles across a star located at the center of a pixel. The expected faintness of the asteroid and the requirement to limit the exposure time to a value compatible with the tracking performances of the spacecraft led us to use the F21 filter, which combines a re-focusing plate for the visible and for the near-infrared, effectively defining a "clear" filter. The system throughput in this configuration is shown in Fig. 2: it defines a very broadband centered at $618 \mathrm{~nm}$ with a $F W H M$ of $460 \mathrm{~nm}$. The central wavelenth is thus close to that of the $R$ filter of the Johnson-Kron-Cousins photometric system $(647 \mathrm{~nm})$, favoring the transformation of the instrumental magnitudes in $R$ band magnitudes.

The observations started on 11.0059 March 2006 UT and ended on 11.9934 UT, thus covering an overall time span of $24 \mathrm{~h}$. The geometric parameters varied only slightly over this interval: the heliocentric distance changed from 2.297 to 2.299 AU, the distance to the Rosetta spacecraft from 1.0606 to $1.0594 \mathrm{AU}$, and the solar phase angle decreased from 41.83 to $41.59^{\circ}$. We obtained a total of 238 images, one image every $6 \mathrm{~min}$, with an exposure time of $300 \mathrm{~s}$ each. Each image corresponds to a window of $512 \times 256$ pixels, centered approximately on the expected position of Steins. The spacecraft tracking was very accurate, and the differential motion between the stars and the asteroid much smaller than the PSF of the camera within a single 


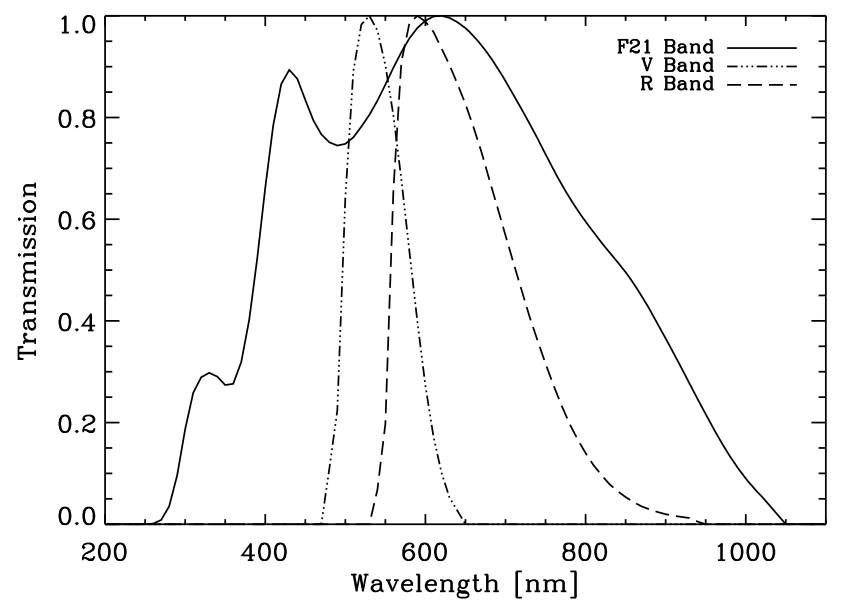

Fig. 2. System throughput of the OSIRIS NAC for the $F 21$ clear filter, including all the optical components, and the quantum efficiency of the CCD. The cut-off at near-ultraviolet wavelengths is due to the near-IR focus plate becoming opaque, the cut-off in the near-IR results from the decreasing quantum efficiency of the CCD. The transmissions of the $V$ and $R$ bands of the Johnson-Kron-Cousin photometric system are overplotted.

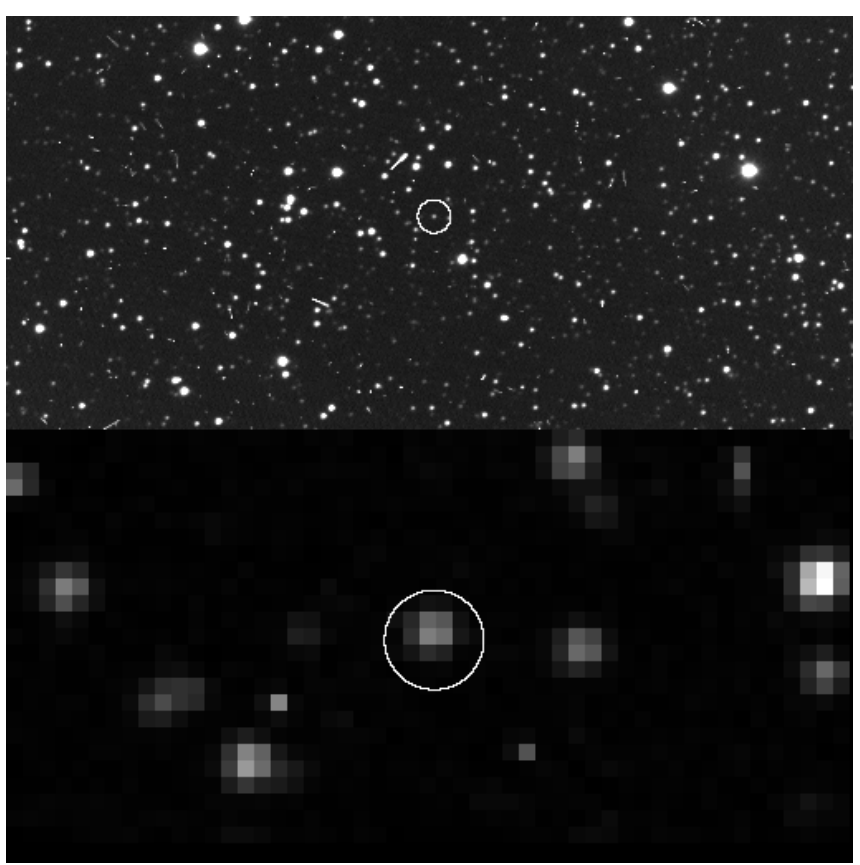

Fig. 3. A full-frame image obtained with the OSIRIS narrow angle camera on 11.2559 March 2006. Asteroid 2867 Steins is highlighted by the circle. An enlarged sub-frame centered on Steins is displayed illustrating the excellent pointing stability of the spacecraft.

exposure (Fig. 1). Figure 3 displays one full image and an enlargement centered on the asteroid.

\section{Data reduction}

\subsection{Image analysis}

The data were reduced with the OSIRIS standard calibration pipeline, in which the following corrections have been implemented (Küppers et al. 2007):

- The CCD is read out using a dual 14 bit analogue-to-digital converter (ADC). DN values created by the "upper" ADC (DN values $\geq 2^{14}$ ) get an additional offset added by the readout electronics. This offset is subtracted.

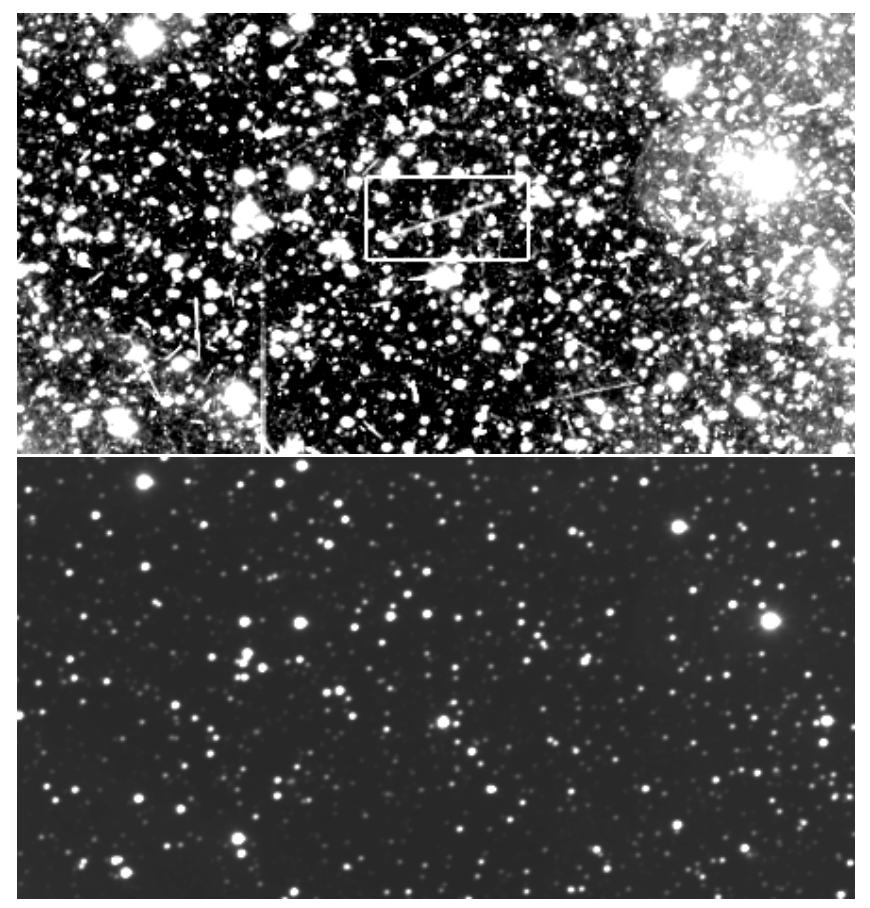

Fig. 4. Top: image of the sum of the individual 238 co-registered images where the asteroid appears as a faint linear track in the white box. Other tracks result from cosmic ray impacts. Bottom: reference background image used to extract the light curve.

- Coherent noise produced by the power converter is filtered out.

- The bias value estimated from bias exposures taken immediately after the Steins observations is subtracted.

- Finally, the images are divided by the flat-field corresponding to the $F 21$ filter combination.

In the course of the observations, the faint asteroid crossed a very rich starfield, getting very close to background stars in several instances. We implemented a rigorous procedure aimed at ensuring that the photometric measurements of the asteroid were properly separated from the contributions of neighboring stars. The first step was to construct a reference background image from the set of 238 images accurately co-registered on the star positions at sub-pixel level. In practice, we used a single reference star free of any artifacts (saturation, impacts of cosmic rays). Figure 4 displays the sum of these 238 co-registered images where the asteroid appears as a faint linear track. The background image itself was constructed by taking the median value of the 238 values at each pixel, thus efficiently removing the asteroid track as well as all artifacts (Fig. 4). The sub-pixel positions of the asteroid were first determined on each individual original image, and then transformed to instantaneous positions on the background image. Circular aperture photometry was performed using the IDL procedure "APER" from the ASTRON library, separately for the 238 original images of the asteroid and for the background image at the corresponding positions. Different aperture radii, $2-5$ pixels were tested and the first value was finally selected as the best compromise between recording the bulk of the flux of the point spread function and limiting the spurious contributions from neighboring stars. Figure 5 illustrates the results of this photometric process, illustrating the importance of the varying contribution of the background, even in the case of the smallest aperture (this effect is even more pronounced with larger apertures). It further shows the presence of outliers, a few 


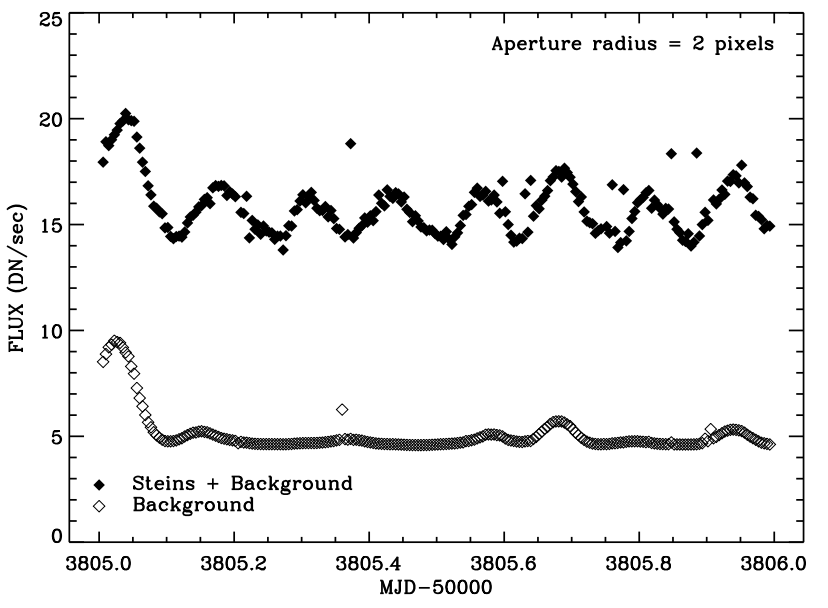

Fig. 5. The raw light curve of asteroid 2867 Steins (filled diamonds) and the contribution from the background (open diamonds).

others being outside the range of the figure. A total of 12 anomalous results were investigated by inspecting enlarged sub-images centered on the asteroid.

A few cases could be readily understood by the presence of very close artifacts (most likely cosmic ray impacts), but the majority of the images looked normal. We then suspected slight variations in the exposure times as the shutter is a very complex mechanism. This question was analyzed by constructing the pixel-to-pixel regressions between each individual image and the background (median) image. Each individual image was first co-registered with the background image, and the effects of cosmic ray impacts were eliminated to avoid biasing the regression. The intercept values do exhibit ramdom variations, typically 1 to $2 \%$, and the corresponding corrections were introduced in the raw photometric measurements of Steins. However, this procedure was unable to correct the remaining outliers, which therefore persist in the final light curve.

\subsection{Uncertainty}

A classical method to globally quantify the uncertainty in the photometric measurements is to study the local noise in a circular annulus $\mathcal{D}_{n}$ centered on, and surrounding the object.

Let $F(i, j)$ be the signal expressed in $\mathrm{DN} \mathrm{s}^{-1}$ at pixel $(i, j)$. The local noise level in the annulus is quantified by the variance of the signal $F(i, j)$ defined by

$\sigma_{F}^{2}=\mathcal{V}^{2}(i, j) \sum_{(i, j) \in \mathcal{D}_{n}}(F(i, j)-\overline{F(i, j)})^{2}$

where $\overline{F(i, j)}$ denotes the mean value of the signal:

$\overline{F(i, j)}=\frac{1}{n_{\mathrm{pix}}} \sum_{(i, j) \in \mathcal{D}_{n}} F(i, j)$.

This method works well when such an annulus can be safely defined (and has in fact been implemented for the photometry of the calibration star, see below) but resulted in values strongly biased by the neighboring stars in the case of asteroid Steins as anticipated.

We therefore turned to a different method, which has been extensively used for the photometry of cometary nuclei observed with the Hubble space telescope, where the presence of asurrounding coma prevents defining the above annulus (e.g., Lamy et al. 2006). The $1 \sigma$ error of the data can be expressed as

$s=\sqrt{\frac{B}{g}+\left(\frac{\mathfrak{R}}{g}\right)^{2}+(f B)^{2}}$

where $B$ is the observed signal in DN, $g=3$ electrons $\mathrm{DN}^{-1}$ is the gain, $\mathfrak{R}=7$ electrons is the readout noise, and $f=0.01$ expresses the flat-field noise as a fraction of the signal.

\subsection{Absolute calibration}

Acccording to the well-known formalism of point-source photometry, the instrumental (or pseudo) magnitude $\bar{m}$ is calculated from the flux $F$ measured on the CCD images (and often expressed in DN/s) according to:

$\bar{m}=-2.5 \log F$.

The zero point $Z P$ of the photometric transformation between the standard magnitude $m$ and the instrumental magnitude for a given aperture (the aperture in which $F$ was calculated) is given by:

$Z P=m-\bar{m}=m+2.5 \log F$

and is independently determined using appropriate reference stars.

The solar spectral analog 16 Cyg B has been observed with the F21 filter as part of the in-flight calibration of the NAC, and photometric measurements have been performed likewise for asteroid Steins using the same aperture i.e., a radius of 2 pixels. The corresponding zero points $Z P_{2}$ for the standard Johnson-Kron-Cousins (JKC) $V$ and $R$ bands are respectively:

$Z P_{2}(V)=19.077 \pm 0.015$

$Z P_{2}(R)=18.727 \pm 0.015$.

The above zero points must now be corrected for the effect of the (red) color of asteroid Steins, especially since the $F 21$ filter is extremely broad, extending from 300 to $1000 \mathrm{~nm}$ (Fig. 2). This is performed according to the procedure outlined below.

Since the spectrum of $16 \mathrm{Cyg} \mathrm{B}$ is well approximated by a solar spectrum $F_{\odot}(\lambda)$, its standard magnitudes $(V, R)$ are given by:

$m_{16 \text { Суg в }}=-2.5 \times \log k_{m} \int_{V, R} F_{\odot}(\lambda) \mathrm{d} \lambda$

where $k_{m}$ is a scaling factor ensuring the proper relationship between spectral flux and magnitude for a given spectral band ( $V$ or $R$ ), and where it implicitly understood that the integral is calculated over the corresponding spectral band.

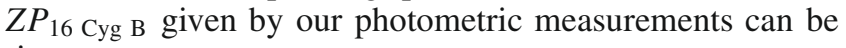
rewritten:

$Z P_{16 \text { Cyg в }}=m_{16 \text { Cyg В }}+2.5 \times \log k_{m}^{\prime} \int_{F 21} F_{\odot}(\lambda) \mathrm{d} \lambda$

where now $k_{m}^{\prime}$ is a scaling coefficient, which ensures the validity of the above equation for the $F 21$ spectral band. It is equivalent to a sensitivity relating $\mathrm{DN} / \mathrm{s}$ to the unit of $F_{\odot}(\lambda)$, i.e., $\operatorname{erg~s}{ }^{-1} \mathrm{~cm}^{-2} \AA^{-1}$.

Similar equations can be written for asteroid Steins:

$m_{\text {Steins }}=-2.5 \times \log k_{m} \int_{V, R} S_{\text {Steins }}(\lambda) \mathrm{d} \lambda$

$Z P_{\text {Steins }}=m_{16 \text { Сyg B }}+2.5 \times \log k_{m}^{\prime} \int_{F 21} S_{\text {Steins }}(\lambda) \mathrm{d} \lambda$ 


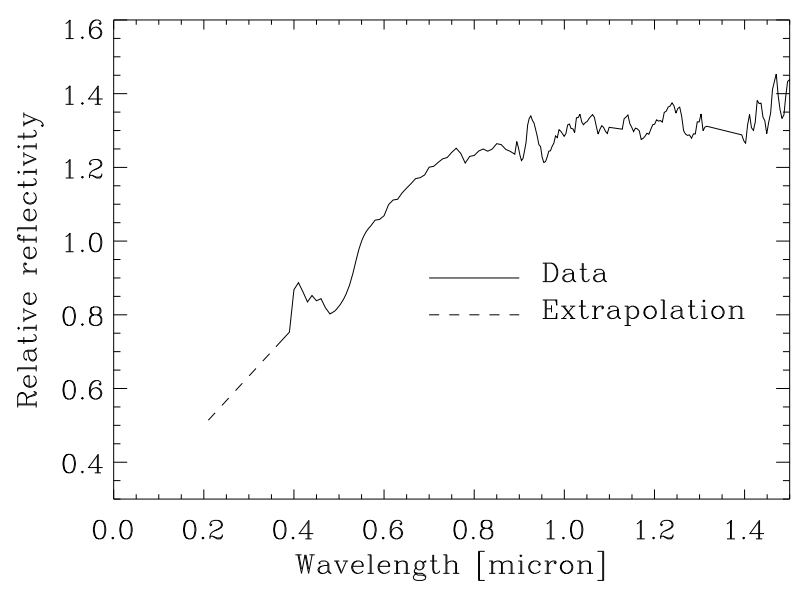

Fig. 6. Relative spectral reflectivity of asteroid 2867 Steins: data from Barucci et al. (2005) and adopted extrapolation below $390 \mathrm{~nm}$.

where:

$S_{\text {Steins }}(\lambda)=R_{\text {Steins }}(\lambda) F_{\odot}(\lambda)$

and $R_{\text {Steins }}(\lambda)$ is the spectral reflectivity of Steins.

By combining the above equations to eliminate the unknowns, we find:

$Z P_{\text {Steins }}=Z P_{16 \text { Cyg B }}+\Delta_{Z P}$

where:

$\Delta_{Z P}=-2.5 \times \log \frac{\int_{V, R} S_{\text {Steins }}(\lambda) \mathrm{d} \lambda \times \int_{F 21} F_{\odot}(\lambda) \mathrm{d} \lambda}{\int_{V, R} F_{\odot}(\lambda) \mathrm{d} \lambda \times \int_{F 21} S_{\text {Steins }}(\lambda) \mathrm{d} \lambda}$.

The spectrum of Steins was taken from Barucci et al. (2005) and extrapolated below $390 \mathrm{~nm}$ where no data is available (Fig. 6); this extrapolation is not critical as the corresponding contribution to the flux measured through the F21 filter is small. Note that only a relative spectrum is required in the above procedure. The F21 spectral band is naturally defined by the corresponding throughput (Fig. 2). The spectral profiles of the $V$ and $R$ bands are taken from Landolt (1992). The solar spectrum $F_{\odot}(\lambda)$ is taken from Vernazza et al. (1976). The colorcorrected zero points appropriate to asteroid Steins are given by:

$$
\begin{aligned}
& Z P_{\text {Steins }}(V)=Z P_{16 \text { Cyg B }}(V)+0.093 \\
& Z P_{\text {Steins }}(R)=Z P_{16 \text { Cyg B }}(R)-0.091 .
\end{aligned}
$$

\section{Results}

\subsection{Light curve}

The apparent $R$ magnitudes of asteroid 2867 Steins in the Johnson-Kron-Cousins (JKC) photometric system are plotted as a function of time in Fig. 7, and reveal a well-defined, slightly asymmetric light curve, indicating that we were seeing the varying cross-section of an elongated body. The light curve has a mean value of $m_{R}=16.07 \pm 0.03$ and an amplitude of $0.25 \pm$ $0.04 \mathrm{mag}$. The corresponding mean magnitude at heliocentric and geocentric distances of $1 \mathrm{AU}$ is therefore $m_{R}=14.13 \pm$ 0.03 , corresponding to a phase angle of $41.71^{\circ}$. The measured magnitudes $R(1,1, \alpha)$ are reported in Table 3 (available online).

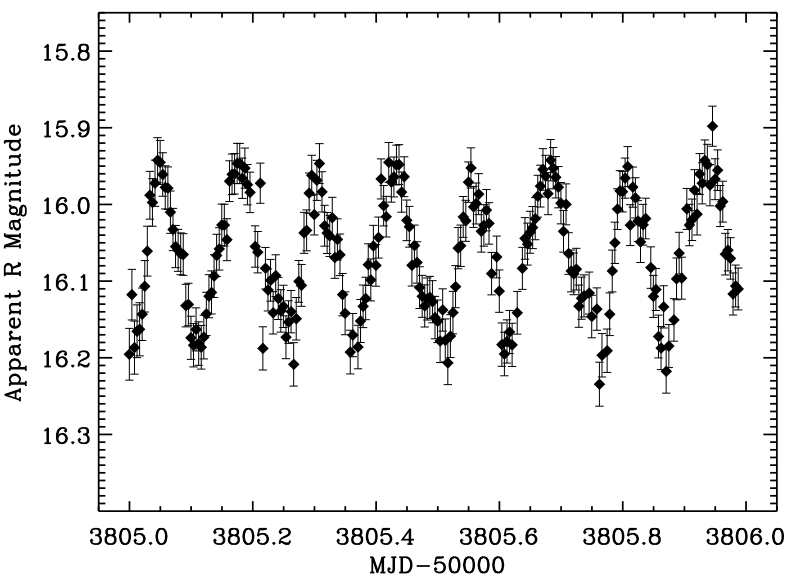

Fig. 7. Apparent $R$ magnitude of asteroid 2867 Steins extracted from the OSIRIS images acquired between 11.006 and 11.993 March 2006 UT.

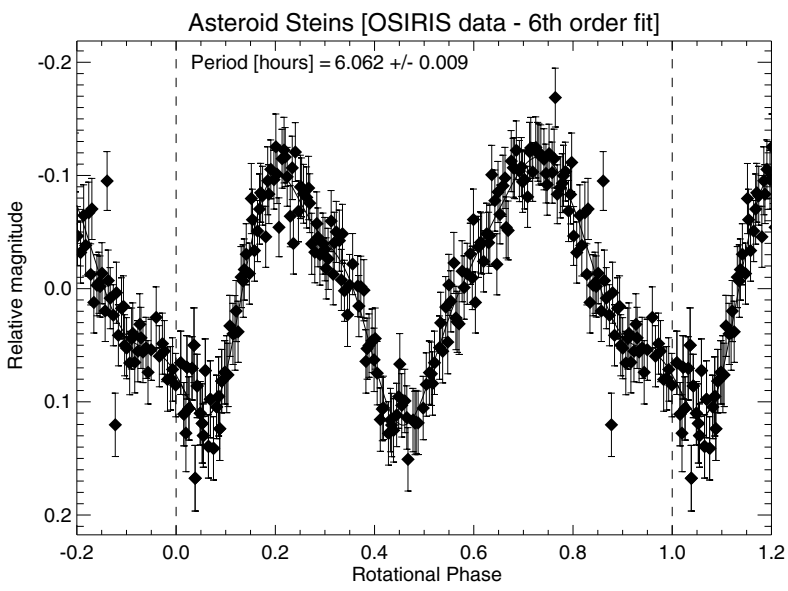

Fig. 8. Folded light curve of asteroid 2867 Steins with a rotational period of $6.059 \mathrm{~h}$. The relative magnitudes and the 6th order Fourier fit are plotted as functions of the rotational phase.

\subsection{Period analysis}

Assuming a double-peaked light curve, we applied different classical period-searching methods to the OSIRIS data.

1. The Fourier analysis, which involves fitting a $n$ th-order Fourier series to the relative magnitudes (Harris et al. 1989b); the resulting folded light curve is displayed in Fig. 8.

2. The Lomb-Scargle method (Lomb 1976; Scargle 1982).

3. The period dispersion minimization method (PDM, Stellingwerf 1978).

4. The WindowCLEAN method (Belton \& Gandhi 1988).

Note that except for the Fourier method, for which a direct estimate of the error bar can be obtained, the calculation of the error bar with the other methods would require the implementation of a Monte Carlo scheme for each of them. We estimate a global error bar from the rms of the values calculated with the different methods, assigning each of them the same statistical weighting. The synodic rotational periods determined with the different methods listed in Table 1, and range from 6.051 to $6.059 \mathrm{~h}$ with a mean of $P_{\text {rot }}=6.054 \mathrm{~h}$ and $\sigma=0.003 \mathrm{~h}$. Table 1 also lists past determinations of the rotational period and the period from our companion Article II (Lamy et al. 2008a), which presents a 
Table 1. Period determinations.

\begin{tabular}{cc}
\hline \hline Method & $P_{\text {rot }}(\mathrm{h})$ \\
\hline Fourier & $6.059 \pm 0.011$ \\
Lomb-Scargle & 6.052 \\
PDM & 6.054 \\
WindowCLEAN & 6.051 \\
Hicks et al. (2004) & $6.060 \pm 0.050$ \\
Küppers et al. (2007) & $6.052 \pm 0.007$ \\
Weissman et al. (2007) & $6.048 \pm 0.007$ \\
& \\
Lamy et al. (2008a) & $6.04681 \pm 0.00002^{1}$ \\
\hline
\end{tabular}

${ }^{1}$ Sidereal period.

solution based on 27 light curves spanning a time interval of more than 2 years, thus leading to the most accurate determination of the sidereal period of $6.04681 \pm 0.00002 \mathrm{~h}$. The difference between the sidereal and the mean synodic period derived from the OSIRIS data amounts to $0.007 \mathrm{~h}$ is compatible with the theoretical difference between both periods at the time of the OSIRIS observations, taking into account the uncertainties.

\subsection{Color}

Broadband color indices of asteroid 2867 Steins have been reported by several groups and are summarized in Table 2. Note that there are typographical errors in the previously reported colors in Küppers et al. (2007). We derived the $V-R$ color index from the reflectivity spectrum of Steins (Fig. 6) in a two-step procedure. First, we calculated the index of the Sun from $F_{\odot}(\lambda)$, and derived a correction by imposing that $(V-R)_{\odot}$ be equal to 0.35 , the standard solar value in the JKC photometric system. This is required to eliminate inherent discrepancies that persist when relating photometric and spectrometric systems. We then applied this correction to the calculated index of Steins and obtained $(V-R)=0.53$. In order to test the robustness of this determination, we repeated the calculation with the Bessel $R$ filter (Bessel 1990), which is widely used for ground-based observations, and found $(V-R)=0.52$. Taking into account the errors on the spectral measurements and calibration, we estimate that the uncertainty on the above determination amounts to 0.03 . Therefore, all available colors agree with each other at the $1.33 \sigma$ level, although differences may be indicative of surface inhomogeneities. As evidenced in the following section, merging $V$ and $R$ magnitudes is however best achieved by taking $(V-R)=0.51$. As a matter of comparison, the reference E-type asteroid 64 Angelina has color indices: $(B-V)=0.72$ to 0.77 and $(V-R)=0.45$ to 0.47 (Rosenbush et al. 2005). To further compare with another E-type asteroid 620 Drakonia for which 2003 reported a color $(V-R)_{\mathrm{J}}=0.56 \pm 0.04$ in the Johnson photometric system, we calculated this index from the reflectivity spectrum of Steins as described above, and found 0.74. Therefore, 2867 Steins appears slightly redder as compared to the mean E-type population. However, Fornasier et al. (2007) noted that the spectral slope and the presence of the $490 \mathrm{~nm}$ band in its visible spectrum are very similar to another known E-type object belonging to the E[II] subgroup. E-type asteroids, although they constitute a quite small population, exhibit at least three different surface mineralogies, as reported by Clark et al. (2004) and Gaffey and Kelley (2004). We emphasize that it is not possible to classify an object only with photometric colors.
Table 2. Color indices of asteroid 2867 Steins.

\begin{tabular}{cccc}
\hline \hline Observer & $(B-V)$ & $(V-R)$ & $(R-I)$ \\
\hline Hicks et al. (2004) & $0.80 \pm 0.03$ & $0.51 \pm 0.03$ & $0.44 \pm 0.03$ \\
Weissman et al. (2007) & & $0.58 \pm 0.03$ & $0.44 \pm 0.03$ \\
This work & & $0.52 \pm 0.03$ & \\
\hline
\end{tabular}

\subsection{Phase function and absolute magnitude}

Among the 27 data sets assembled in our Article II to reconstruct the shape of asteroid Steins, only 9, beside our OSIRIS data set, are calibrated and of sufficient photometric quality to investigate the phase function of asteroid 2867 Steins. Seven of them are expressed in $R$ magnitudes; the other two are $V$ magnitudes that we transformed to $R$ using the above color index as giving the best overall agreement. The minimum and maximum magnitudes of the eight light curves, reduced to heliocentric and geocentric distances of $1 \mathrm{AU}$ are plotted versus solar phase angle in Fig. 9. The uncertainties affecting the various measurements are not plotted for clarity but listed at each date.

For an irregular body possibly having an inhomogeneous surface, the definition of the disk-integrated phase function becomes ambiguous ( $\mathrm{Li}$ et al. 2004). This is because the observed disk-integrated reflectivity depends upon the solar phase angle on the one hand, but also on what part of the surface is being seen on the other hand, the latter being determined by the pole orientation and the rotational phase. Therefore the simplest way to construct a phase function is to use characteristic points of the rotational light curves such as the maxima, the means, or the minima. Theoretical simulations performed by Li et al. (2004) have shown that the first two alternatives are preferable, and we decided to use the maxima for the analysis of the phase function as we already did for the nucleus of comet 67P/ChuryumovGerasimenko (Lamy et al. 2007). A linear phase law fits the maxima extremely well, and taking into account the uncertainties of the various data sets, the phase coefficient $\beta$ can be strongly constrained to $\beta=0.025 \pm 0.001 \mathrm{mag} / \mathrm{deg}$, and this leads to an absolute $R$ magnitude of the observed largest cross-section of the asteroid $R(1,1,0)_{\max }=12.97 \pm 0.02$. With the exception of two sets, a similar phase law fits the minima well leading to $R(1,1,0)_{\min }=13.22 \pm 0.03$. However we cannot exclude a more complex behavior where the phase coefficient would vary with the phase angle, especially if a larger $V-R$ color index is adopted. Then the slope would be shallower below $\sim 30^{\circ}$, and would steepen beyond to match the OSIRIS data. Such a behavior prevails for some asteroids, and has been measured on meteorite powders (Nakamura et al. 2002).

We next attempted to implement the IAU-recommended $H-G$ formalism (Bowell et al. 1989) to estimate an expected opposition effect. Since we have no observation at phase angles below $7^{\circ}$, the fit is solely constrained by the quasi-linear part of the phase function. The maximum values are compatible with $G=0.35 \pm 0.05$, and the resulting opposition surge is $\sim 0.25$ mag. The mean value of $H$ is $12.84 \pm 0.05$ with extreme values of $12.71 \pm 0.05$ for the maximum and $12.96 \pm 0.05$ for the minimum cross-sections.

Harris et al. (1989a) investigated the phase function of two E-type asteroids, 44 Nysa and 64 Angelina. After proper scaling, the two curves were indistinguishable, and characterized by a linear part with a phase coefficient of $0.022 \mathrm{mag} / \mathrm{deg}$, an amplitude of the opposition effect of $0.25 \mathrm{mag}$ (taken at $\alpha=0.1^{\circ}$ and relative to the extrapolation of the linear part) and $G=0.51$. When these results are compared with ours for 2867 Steins, it should be kept in mind that the ground-based observations are 


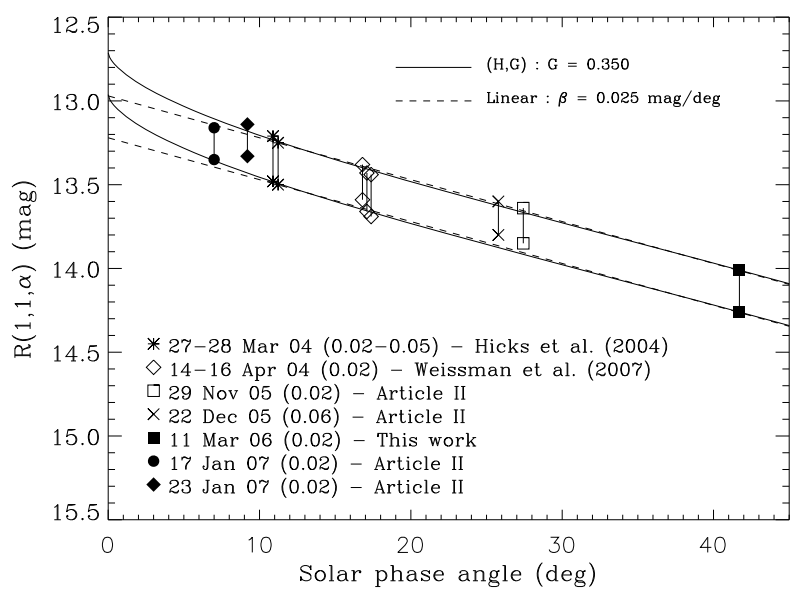

Fig. 9. Phase function of asteroid 2867 Steins derived from 10 ligthcurves. The extrema for each data set represent the minima and the maxima of the ligthcurves. The best fit linear relationship (dashed line) and the curve calculated with the $H-G$ formalism (solid line) are overplotted. The $1 \sigma$ uncertainty affecting the various measurements are indicated in brackets.

limited to a maximum phase angle of $\sim 30^{\circ}$, an angular domain where the Steins phase function could be shallower as discussed above, and therefore more in line with the results of Harris et al. (1989). Belskaya \& Shevchenko (2000) performed a systematic study of 33 asteroids, including the above two, and two additional E-type asteroids, 214 Aschera and 317 Roxane. The phase functions of the four objects are identical, and have properties very distinct from the other classes of asteroids, in addition to their large albedo. This is well illustrated by their Figs. 3 and 4 where they plotted the parameters of the phase function versus albedo. They further derived a linear relationship between the phase coefficient (or slope) of the linear part (denoted "b") and the logarithm of the visible albedo $p_{\mathrm{V}}$. With a slope $b=0.025 \pm 0.001 \mathrm{mag} / \mathrm{deg}$ and $p_{\mathrm{V}}(0)=0.34 \pm 0.06$ (our Article III), 2867 Steins does line up very well with this relationship, but closer to the group of S- than the E-type objects (Fig. 10). Belskaya et al. (2003) incorporated one additional E-type asteroid, 620 Drakonia, in their analysis and presented a synthetic comparison of the photometric properties between $\mathrm{S}$ and E-type objects (their Table 3). While spectral observations of 2867 Steins (Barucci et al. 2005) and the similarity of its emissivity spectrum to the enstatite meteorites support its E-type classification (Article IV), it appears that its photometric properties (phase function, albedo) are somehow intermediate between $\mathrm{S}$ and E-types. Further noting its red color altogether makes asteroid 2867 Steins a rather peculiar and intringuing object.

\section{Summary}

We have presented an in-depth photometric analysis of the observations of the Rosetta mission flyby target asteroid 2867 Steins obtained with the OSIRIS narrow angle camera onboard the Rosetta spacecraft. Our analysis includes an accurate absolute calibration using a solar analog star and a quantification of the uncertainties. Further combining with ground-based observations allowed us to ascertain the photometric properties of this asteroid, and to discuss their implication for its taxonomic classification. Our main conclusions are as follows:

We measured a mean apparent $R$ band magnitude in the JKC photometric system of $16.07 \pm 0.03$ and an amplitude of $0.25 \pm 0.04 \mathrm{mag}$. The rotational light curve is slightly

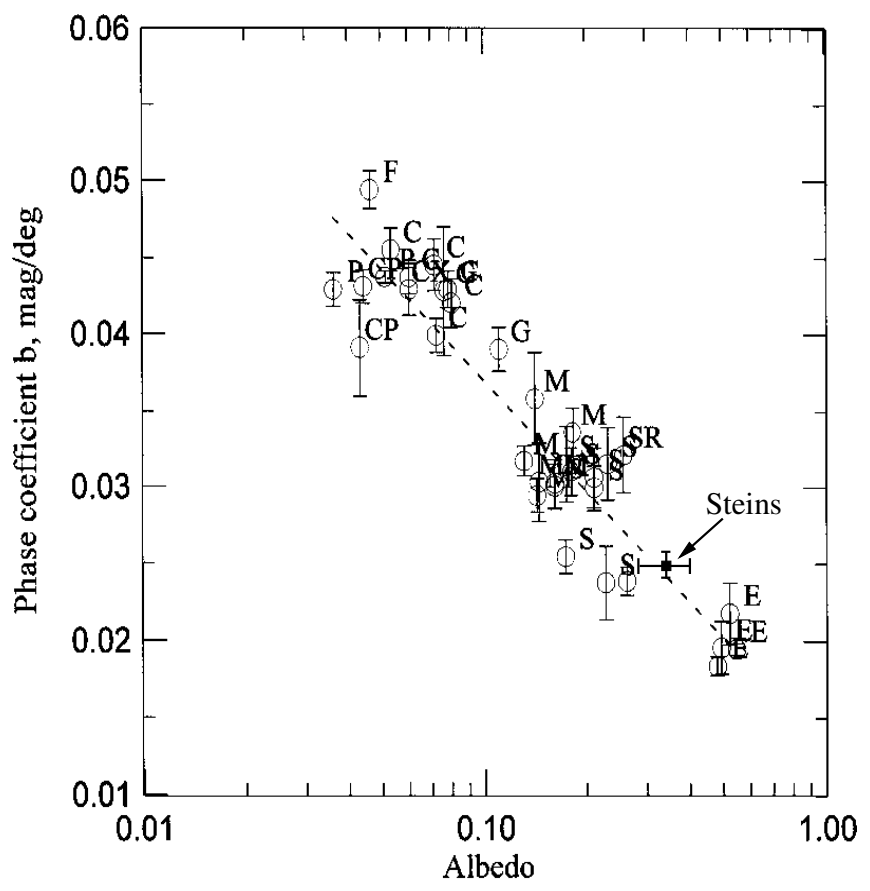

Fig. 10. Dependence of the slope "b" of the linear part of the phase function on geometric albedo for asteroids of different types, as compiled by Belskaya \& Shevchenko (2000, their Fig. 4). We have superimposed our result for Steins: the slope from this work and the geometric albedo from Article III.

asymmetric. Periodocity analysis using three methods converge to a synodic rotation period of $6.054 \pm 0.003 \mathrm{~h}$ at the time of the OSIRIS observations. Combining presently available observations that cover a range of solar phase angle from 7 to $42^{\circ}$ allows us to fit a linear phase law with a phase coefficient $\beta=0.025 \pm$ 0.001 . This leads to a reduced $R(1,1,0)$ ranging from $12.97 \pm$ 0.02 (maximum cross-section) to $13.22 \pm 0.03 \mathrm{mag}$ (minimum cross-section). Fitting a more elaborate phase function in terms of the $H-G$ formalism is much less constrained because of the lack of data at phase angle less than $7^{\circ}$. The best fit parameters are $G=0.035 \pm 0.005$ and $H=12.71 \pm 0.05$ (maximum cross-section) to $12.96 \pm 0.05$ (minimum cross-section). Further photometry data within the opposition surge region is needed to ascertain the validity of the $H-G$ description, and the value of the opposition surge. While the similarity of the emissivity spectrum of 2867 Steins to the enstatite meteorites firmly supports its E-type classification, its photometric properties (phase function, color, albedo) are somehow intermediate between S- and E-types making this object rather peculiar and intringuing.

Acknowledgements. OSIRIS was built by a consortium of the MaxPlanck-Institut für Sonnensystemforschung, Lindau, Germany, the Laboratoire d'Astrophysique de Marseille, France, the Centro Interdipartimentale Studi e Attivita' Spaziali, University of Padova, Italy, the Instituto de Astrofisica de Andalucia, Granada, Spain, the Research and Scientific Support Department of the European Space Agency (ESA/ESTEC), Noordwijk, The Netherlands, the Instituto Nacional de Tecnica Aerospacial, Madrid, Spain, the Institut für Datentechnik und Kommunikationsnetze der Technischen Universitat, Braunschweig and the Department of Astronomy and Space Physics of Uppsala University, Sweden. The support of the national funding agencies DLR, CNES, ASI, MEC, NASA, and SNSB is gratefully acknowledged. S. Lowry aknowledges support from the Leverhulme Trust. The Rosetta spacecraft is operated by the Rosetta Science Operations Centre at ESA/ESTEC and by the Rosetta Mission Operations Centre at ESA/ESOC. A portion of this work was performed at the Jet Propulsion Laboratory under contract with NASA. JPL's Horizons 
online ephemeris generator was used to calculate the asteroid's position and rate of motion.

\section{References}

Barucci, M. A., Fulchignoni, M., Fornasier, S., et al. 2005, A\&A, 430, 313 Barucci, M. A., Fornasier, S., Dotto, E., et al. 2008, A\&A, 477, 665 (Article IV) Belskaya, I. N., \& Shevchenko, V. G. 2000, Icarus, 147, 94

Belskaya, I. N., Shevchenko, V. G., Kiselev, N. N., et al. 2003, Icarus, 166, 276

Belton, M. J. S., \& Gandhi, A. 1988, BAAS, 20, 836

Bessel, M. S. 1990, PASP, 102, 1181

Bowell, E., Hapke, B., Domingue, D., et al. 1989, Application of Photometric Models to Asteroids, in Asteroids II, ed. R. P. Binzel, T. Gehrels, \& M. D. Matthews (Tucson: Univ. of Arizona press), 524

Calvel, B., Castel, D., Lamy, P. L., Dohlen, K., \& Bougoin, M. 1999, Proc. SPIE, 3785,56

Clark, B. E., Bus, S. J., Rivkin, A. S., et al. 2004, E-Type asteroid spectroscopy and compositional modeling, J. Geophys. Res., 109, Issue E2, CiteID E0200

Dohlen, K., Saisse, M., Claeysen, G., Lamy, P. L., \& Boit, J.-L. 1996, Optic. Eng., 35, 1150

Fornasier, S., Belskaya, I., Fulchignoni, M., et al. 2006, A\&A, 449, L9

Fornasier, S., Migliorini, A., Dotto, E., \& Barucci, M. A. 2007, in preparation
Gaffey, M. J., \& Kelley, M. S. 2004 LPSC Abstr., 1812

Harris, A. W., Young, J. W., Contreiras, L., et al. 1989a, Icarus, 81, 365 Harris, A. W., Young, J. W., Bowell, E., et al. 1989b, Icarus, 77, 171

Hicks, M. D., Bauer, J. M., \& Tokunaga, A. T. 2004, IAU Circular, 8315

Keller, H. U., Barbieri, C., Lamy, P. L., et al. 2007, Space Sci. Rev., 128, 433

Küppers, M., Mottola, S., Lowry, S. C., et al. 2006, A\&A, 462, L13

Lamy, P. L., Toth, I., Weaver, H. A., et al. 2006, A\&A, 458, 669

Lamy, P. L., Toth, I., Davidsson, B. J. R., et al. 2007, Space Sci. Rev., 128, 74

Lamy, P. L., Kaasalainen, M., Lowry, S., et al. 2008a, A\&A, 487, 1179 (Article II)

Lamy, P. L., Jorda, L., Fornasier, S., et al. 2008b, A\&A, 487, 1187 (Article III) Landolt, A. U. 1992, AJ, 104, 340

Li, J.-Y., A'Hearn, M. F., \& McFadden, L. A. 2004, Icarus, 172, 415

Lomb, N. R. 1976, Ap\&SS, 39, 447

Lowry, S. C., Fitzsimmons, A., Jorda, L., et al. 2006, BAAS, 38, 492

Nakamura, A. M., Kamei, A., \& Minato, T. 2002, in Mem. Soc. Astron. Ital., 73, 722

Rosenbush, V. K., Kiselev, N. N., Shevchenko, V. G., et al. 2005, Icarus, 178, 222

Scargle, J. D. 1982, ApJ, 263, 835

Stellingwerf, R. F. 1978, ApJ, 224, 953

Vernazza, J. E., Avrett, E. H., \& Loeser, R. 1976, ApJS, 30, 1

Weissman, P. R., Lowry, S. C., \& Choi, Y. J. 2007, A\&A, 466, 737 
L. Jorda et al.: Asteroid Steins. I., Online Material p 1

Table 3. Steins $R(1,1, \alpha)$ magnitudes with $1-\sigma$ error bars.

\begin{tabular}{|c|c|c|}
\hline$\overline{\mathrm{MJD}}(\mathrm{UTC})^{a}$ & $\overline{R(1,1, \alpha)}$ & $\overline{\bar{\sigma}}$ \\
\hline 53804.99981 & 14.261 & 0.033 \\
\hline 53805.00396 & 14.183 & 0.033 \\
\hline 53805.00813 & 14.252 & 0.035 \\
\hline 53805.01230 & 14.231 & 0.034 \\
\hline 53805.01646 & 14.228 & 0.034 \\
\hline 53805.02063 & 14.209 & 0.034 \\
\hline 53805.02480 & 14.172 & 0.034 \\
\hline 53805.02896 & 14.127 & 0.032 \\
\hline 53805.03313 & 14.054 & 0.031 \\
\hline 53805.03730 & 14.063 & 0.031 \\
\hline 53805.04146 & 14.038 & 0.030 \\
\hline 53805.04563 & 14.008 & 0.029 \\
\hline 53805.04980 & 14.011 & 0.028 \\
\hline 53805.05396 & 14.027 & 0.028 \\
\hline 53805.05813 & 14.043 & 0.028 \\
\hline 53805.06230 & 14.044 & 0.028 \\
\hline 53805.06646 & 14.076 & 0.028 \\
\hline 53805.07063 & 14.098 & 0.028 \\
\hline 53805.07480 & 14.121 & 0.028 \\
\hline 53805.07896 & 14.126 & 0.027 \\
\hline 53805.08313 & 14.130 & 0.027 \\
\hline 53805.08730 & 14.131 & 0.027 \\
\hline 53805.09146 & 14.198 & 0.028 \\
\hline 53805.09563 & 14.196 & 0.028 \\
\hline 53805.09980 & 14.240 & 0.028 \\
\hline 53805.10396 & 14.249 & 0.028 \\
\hline 53805.10813 & 14.229 & 0.028 \\
\hline 53805.11230 & 14.247 & 0.028 \\
\hline 53805.11646 & 14.252 & 0.028 \\
\hline 53805.12063 & 14.239 & 0.028 \\
\hline 53805.12480 & 14.209 & 0.028 \\
\hline 53805.12896 & 14.185 & 0.028 \\
\hline 53805.13313 & 14.181 & 0.028 \\
\hline 53805.13730 & 14.159 & 0.028 \\
\hline 53805.14146 & 14.132 & 0.028 \\
\hline 53805.14563 & 14.124 & 0.028 \\
\hline 53805.14980 & 14.093 & 0.027 \\
\hline 53805.15396 & 14.093 & 0.027 \\
\hline 53805.15813 & 14.112 & 0.027 \\
\hline 53805.16230 & 14.035 & 0.027 \\
\hline 53805.16646 & 14.027 & 0.026 \\
\hline 53805.17063 & 14.026 & 0.026 \\
\hline 53805.17480 & 14.012 & 0.026 \\
\hline 53805.17896 & 14.012 & 0.026 \\
\hline 53805.18313 & 14.032 & 0.026 \\
\hline 53805.18730 & 14.018 & 0.026 \\
\hline 53805.19146 & 14.040 & 0.026 \\
\hline 53805.19563 & 14.050 & 0.026 \\
\hline 53805.20396 & 14.121 & 0.027 \\
\hline 53805.20813 & 14.128 & 0.027 \\
\hline 53805.21230 & 14.038 & 0.026 \\
\hline 53805.21646 & 14.254 & 0.028 \\
\hline 53805.22063 & 14.149 & 0.027 \\
\hline 53805.22480 & 14.177 & 0.027 \\
\hline 53805.22896 & 14.165 & 0.027 \\
\hline 53805.23313 & 14.207 & 0.028 \\
\hline 53805.23730 & 14.159 & 0.027 \\
\hline 53805.24146 & 14.188 & 0.027 \\
\hline 53805.24563 & 14.205 & 0.028 \\
\hline 53805.24980 & 14.199 & 0.027 \\
\hline 53805.25396 & 14.239 & 0.028 \\
\hline 53805.25813 & 14.219 & 0.028 \\
\hline 53805.26230 & 14.206 & 0.028 \\
\hline 53805.26646 & 274 & 0.028 \\
\hline
\end{tabular}

Table 3. continued.

\begin{tabular}{lcc}
\hline MJD (UTC) & $R(1,1, \alpha)$ & $\sigma$ \\
\hline 53805.27063 & 14.215 & 0.028 \\
53805.27480 & 14.166 & 0.027 \\
53805.27896 & 14.171 & 0.027 \\
53805.28313 & 14.103 & 0.027 \\
53805.28730 & 14.100 & 0.027 \\
53805.29146 & 14.051 & 0.026 \\
53805.29563 & 14.028 & 0.026 \\
53805.29980 & 14.079 & 0.027 \\
53805.30396 & 14.034 & 0.026 \\
53805.30813 & 14.013 & 0.026 \\
53805.31230 & 14.050 & 0.026 \\
53805.31646 & 14.094 & 0.027 \\
53805.32063 & 14.103 & 0.027 \\
53805.32480 & 14.107 & 0.027 \\
53805.32896 & 14.083 & 0.027 \\
53805.33313 & 14.135 & 0.027 \\
53805.33730 & 14.112 & 0.027 \\
53805.34146 & 14.132 & 0.027 \\
53805.34563 & 14.184 & 0.028 \\
53805.34980 & 14.208 & 0.028 \\
53805.35813 & 14.258 & 0.029 \\
53805.36230 & 14.236 & 0.028 \\
53805.37063 & 14.252 & 0.028 \\
53805.37480 & 14.218 & 0.028 \\
53805.37896 & 14.199 & 0.028 \\
53805.38313 & 14.189 & 0.028 \\
53805.38730 & 14.144 & 0.027 \\
53805.39146 & 14.164 & 0.027 \\
53805.39563 & 14.120 & 0.027 \\
53805.39980 & 14.145 & 0.027 \\
53805.40396 & 14.109 & 0.028 \\
53805.40813 & 14.033 & 0.026 \\
53805.41230 & 14.068 & 0.026 \\
53805.41646 & 14.082 & 0.027 \\
53805.42063 & 14.011 & 0.026 \\
53805.42480 & 14.037 & 0.026 \\
53805.42896 & 14.030 & 0.026 \\
53805.43313 & 14.015 & 0.026 \\
53805.43730 & 14.014 & 0.026 \\
53805.44146 & 14.050 & 0.026 \\
53805.44563 & 14.030 & 0.026 \\
53805.44980 & 14.087 & 0.026 \\
53805.45397 & 14.095 & 0.027 \\
53805.45813 & 14.145 & 0.027 \\
53805.46230 & 14.120 & 0.027 \\
53805.46647 & 14.142 & 0.027 \\
53805.47063 & 14.174 & 0.027 \\
53805.47480 & 14.186 & 0.027 \\
53805.47897 & 14.198 & 0.027 \\
53805.48313 & 14.189 & 0.027 \\
53805.48730 & 14.187 & 0.027 \\
53805.49147 & 14.193 & 0.027 \\
53805.49563 & 14.214 & 0.028 \\
53805.49980 & 14.219 & 0.028 \\
53805.50397 & 14.244 & 0.028 \\
53805.50813 & 14.204 & 0.028 \\
53805.51230 & 14.243 & 0.028 \\
53805.51647 & 14.273 & 0.028 \\
53805.52063 & 14.238 & 0.028 \\
53805.52480 & 14.207 & 0.028 \\
53805.52897 & 14.173 & 0.027 \\
53805.53313 & 14.123 & 0.027 \\
53805.53730 & 14.120 & 0.027 \\
53805.54147 & 14.082 & 0.027 \\
\hline & & \\
\hline
\end{tabular} 5


L. Jorda et al.: Asteroid Steins. I., Online Material $p 2$

Table 3. continued.

\begin{tabular}{lcc} 
& & \\
\hline \hline MJD (UTC) & $R(1,1, \alpha)$ & $\sigma$ \\
\hline 53805.54563 & 14.087 & 0.027 \\
53805.54980 & 14.037 & 0.026 \\
53805.55397 & 14.019 & 0.026 \\
53805.55813 & 14.069 & 0.027 \\
53805.56230 & 14.065 & 0.027 \\
53805.56647 & 14.053 & 0.027 \\
53805.57063 & 14.101 & 0.027 \\
53805.57480 & 14.093 & 0.027 \\
53805.57897 & 14.074 & 0.027 \\
53805.58313 & 14.091 & 0.027 \\
53805.58730 & 14.156 & 0.028 \\
53805.59563 & 14.135 & 0.027 \\
53805.59980 & 14.179 & 0.028 \\
53805.60397 & 14.249 & 0.028 \\
53805.60813 & 14.261 & 0.028 \\
53805.61230 & 14.245 & 0.028 \\
53805.61647 & 14.233 & 0.028 \\
53805.62063 & 14.249 & 0.028 \\
53805.62897 & 14.208 & 0.028 \\
53805.63730 & 14.150 & 0.027 \\
53805.64147 & 14.111 & 0.027 \\
53805.64563 & 14.118 & 0.027 \\
53805.64980 & 14.103 & 0.027 \\
53805.65397 & 14.096 & 0.027 \\
53805.65813 & 14.085 & 0.027 \\
53805.66230 & 14.056 & 0.027 \\
53805.66647 & 14.042 & 0.027 \\
53805.67063 & 14.021 & 0.027 \\
53805.67480 & 14.029 & 0.027 \\
53805.67897 & 14.052 & 0.027 \\
53805.68313 & 14.009 & 0.027 \\
53805.68730 & 14.019 & 0.027 \\
53805.69147 & 14.031 & 0.027 \\
53805.69563 & 14.044 & 0.027 \\
53805.69980 & 14.065 & 0.027 \\
53805.70397 & 14.101 & 0.027 \\
53805.70813 & 14.066 & 0.027 \\
53805.71230 & 14.130 & 0.027 \\
53805.71647 & 14.153 & 0.027 \\
53805.72063 & 14.157 & 0.027 \\
53805.72480 & 14.151 & 0.027 \\
53805.72897 & 14.199 & 0.028 \\
53805.73313 & 14.189 & 0.027 \\
53805.73730 & 14.186 & 0.027 \\
53805.74563 & 14.182 & 0.027 \\
53805.74980 & 14.213 & 0.028 \\
53805.75813 & 14.203 & 0.028 \\
53805.76230 & 14.301 & 0.029 \\
53805.76647 & 14.263 & 0.028 \\
53805.77480 & 14.257 & 0.028 \\
53805.77897 & 14.210 & 0.028 \\
53805.78313 & 14.153 & 0.027 \\
53805.78730 & 14.116 & 0.027 \\
53805.79147 & 14.072 & 0.027 \\
53805.79563 & 14.049 & 0.026 \\
53805.79980 & 14.050 & 0.026 \\
53805.80397 & 14.032 & 0.026 \\
53805.80813 & 14.017 & 0.026 \\
53805.81230 & 14.093 & 0.026 \\
53805.81647 & 14.044 & 0.026 \\
53805.82063 & 14.058 & 0.026 \\
53805.82480 & 14.088 & 0.026 \\
53805.82897 & 14.115 & 0.027 \\
53805.83313 & 14.093 & 0.027 \\
53805.83730 & 14.085 & 0.027 \\
53805.84563 & 14.149 & 0.027 \\
\hline & & \\
53
\end{tabular}

Table 3. continued.

\begin{tabular}{ccc}
\hline \hline MJD (UTC $^{a}$ & $R(1,1, \alpha)$ & $\sigma$ \\
\hline 53805.84980 & 14.186 & 0.027 \\
53805.85397 & 14.177 & 0.027 \\
53805.85813 & 14.239 & 0.028 \\
53805.86230 & 14.254 & 0.028 \\
53805.86647 & 14.200 & 0.028 \\
53805.87063 & 14.284 & 0.028 \\
53805.87480 & 14.251 & 0.028 \\
53805.88313 & 14.217 & 0.028 \\
53805.88730 & 14.164 & 0.027 \\
53805.89147 & 14.130 & 0.027 \\
53805.89564 & 14.163 & 0.027 \\
53805.90397 & 14.072 & 0.027 \\
53805.90813 & 14.093 & 0.027 \\
53805.91230 & 14.086 & 0.027 \\
53805.91647 & 14.048 & 0.027 \\
53805.92064 & 14.079 & 0.027 \\
53805.92480 & 14.027 & 0.027 \\
53805.92897 & 14.039 & 0.027 \\
53805.93313 & 14.009 & 0.027 \\
53805.93730 & 14.015 & 0.027 \\
53805.94147 & 14.041 & 0.027 \\
53805.94564 & 13.964 & 0.026 \\
53805.94980 & 14.034 & 0.027 \\
53805.95397 & 14.022 & 0.026 \\
53805.95814 & 14.069 & 0.027 \\
53805.96230 & 14.063 & 0.027 \\
53805.96647 & 14.131 & 0.027 \\
53805.97064 & 14.126 & 0.027 \\
53805.97480 & 14.137 & 0.027 \\
53805.97897 & 14.183 & 0.027 \\
53805.98314 & 14.173 & 0.027 \\
53805.98730 & 14.177 & 0.027 \\
\hline & &
\end{tabular}

${ }^{a}$ Modified Julian Date. 